\title{
Spin and charge order in doped Hubbard model: long-wavelength collective modes
}

\author{
Chia-Chen Chang and Shiwei Zhang \\ Department of Physics, College of William and Mary, Williamsburg, VA 23187
}

\begin{abstract}
Determining the ground state properties of the two-dimensional Hubbard model has remained an outstanding problem. Applying recent advances in constrained path auxiliary-field quantum Monte Carlo techniques and simulating large rectangular periodic lattices, we calculate the long-range spin and charge correlations in the ground state as a function of doping. At intermediate interaction strengths, an incommensurate spin density wave (SDW) state is found, with antiferromagnetic order and essentially homogeneous charge correlation. The wavelength of the collective mode decreases with doping, as does its magnitude. The SDW order vanishes beyond a critical doping. As the interaction is increased, the holes go from a wave-like to a particle-like state, and charge ordering develops which eventually evolves into stripe-like states.
\end{abstract}

PACS numbers: 71.10.Fd, 02.70.Ss

The Hubbard model [1] is one of the simplest and most fundamental models for quantum many-body systems. Since the discovery of high- $T_{C}$ superconductors in 1986, the twodimensional (2D) repulsive Hubbard model has received great interest, as a potential model for describing the essential physics of the copper-oxygen plane in cuprates [2]. Thanks to intensive analytic and numerical investigations, some aspects of its phase diagram have been understood [3]. For example, at half-filling (one electron per lattice site), the system has long-range antiferromagnetic (AF) order [4, 5, 6, 7]. However, many basic issues still remain unknown or controversial. For instance, what happens to the AF order when the system is doped? This is important not only for understanding the magnetic properties, which the Hubbard model was originally designed to describe, but also in the context of high- $T_{c}$, which shares the same parameter regime and is believed to be closely related to $\mathrm{AF}$ fluctuations.

The difficulty in treating the Hubbard model underscores a more general theme, namely the challenge of accurate treatment of strongly correlated systems. Recent experimental work [8] with cold fermionic atoms in optical lattices offers a promising new avenue - potentially direct simulations of Hubbard-like models with "lattice emulators" [9]. We believe this increases, rather than decreases, the demand on "traditional" numerical simulations. Although all numerical methods have their limitations, high quality data on the Hubbard model will provide guidance and allow direct comparison with experiments, thereby creating a new level of synergy to tackle the problem of strong electron correlations.

There are many earlier investigations of the spin and charge correlations in the 2D Hubbard model [3, 4, 5, 6, 7, 10, 11, 12, 13, 14, 15, 16, 17, 18, 19], but reaching large system sizes with sufficient accuracy has been a challenge. Recently, we have calculated the equation of state from low to intermediate interaction strengths on periodic lattices of up to $\sim 16 \times 16$ [20]. The constrained path Monte Carlo (CPMC) method [21, 22] was generalized to incorporate a boundary condition integration technique [23], which removed short-range finitesize effects. It was found that, immediately upon doping, the thermodynamic stability condition is violated. This implied the existence of a spatially inhomogeneous phase. In the ab- sence of long-range collective modes, the results are an accurate representation of the thermodynamic limit, and the instability would indicate phase separation. On the other hand, if long-range collective modes existed whose characteristic length exceeds the size of the super cell $(\sim 16)$, they would not be fully captured in the simulations. The nature of the AF fluctuation in the doped Hubbard model thus remains to be resolved.

Here we address the question, by employing recent algorithmic advances [20, 24, 25] in CPMC and simulating rectangular supercells on parallel computers. Much larger linear dimension (128) is reached than in previous studies $(\sim 16)$, and detailed measurements are obtained of the spin-spin and charge-charge correlations in the ground state, at intermediate interaction strengths where our method is very accurate. Our results show that long wavelength collective modes of incommensurate spin density wave (SDW) states appear as the system is doped, with AF spin order but essentially homogeneous charge correlation. Charge correlation develops as the interaction is further increased. We quantify the nature of such states, and discuss how they relate to the "stripe" states at large interactions.

The Hamiltonian for the single-band Hubbard model is

$$
H=H_{1}+H_{2}=-t \sum_{\mathbf{j}, \delta, \sigma} c_{\mathbf{j}, \sigma}^{\dagger} c_{\mathbf{j}+\delta, \sigma}+U \sum_{\mathbf{j}} n_{\mathbf{j} \uparrow} n_{\mathbf{j} \downarrow},
$$

where $c_{\mathbf{j}, \sigma}^{\dagger}\left(c_{\mathbf{j}, \sigma}\right)$ creates (annihilates) an electron with spin $\sigma(\sigma=\uparrow, \downarrow)$ at lattice site $\mathbf{j}$, and $\delta$ connects all possible nearest-neighbor sites. The supercell has $N=L_{x} \times L_{y}$ sites. The density is $n \equiv\left(N_{\uparrow}+N_{\downarrow}\right) / N$, where $N_{\sigma}$ is the number of electrons with spin $\sigma$; doping is $h \equiv 1-n$. We implement twist-averaged boundary conditions [23], under which the wave function gains a phase when electrons hop around lattice boundaries: $\Psi\left(\ldots, \mathbf{r}_{j}+\mathbf{L}, \ldots\right)=e^{i \widehat{\mathbf{L}} \cdot \Theta} \Psi\left(\ldots, \mathbf{r}_{j}, \ldots\right)$, where $\widehat{\mathbf{L}}$ is the unit vector along $\mathbf{L}$, and $\Theta=\left(\theta_{x}, \theta_{y}\right)$ are random twist angles over which we average.

The generalized CPMC method [20, 21, 22, 25] used here obtains the many-body ground state by repeated projections with $e^{-\tau H}$ ( $\tau$ is the projection time step), as in standard quantum Monte Carlo (QMC). The two-body part, $e^{-\tau H_{2}}$, is decoupled via the Hubbard-Stratonovich transformation into a 
sum over one-body projectors in Ising fields [4]. The projection is then realized efficiently by importance-sampled random walks with non-orthogonal Slater determinants (SDs), where the one-body projectors propagate one SD into another, and the many-dimensional sum over Ising fields is performed by Monte Carlo. The usual fermion sign/phase problem is controlled approximately by a global phase condition on the SDs. This is the only approximation in the method. The basic idea of the approximation is as follows. The many-body ground state is given by $\left|\Psi_{0}\right\rangle=\sum_{\phi} w(\phi)|\phi\rangle$, where $|\phi\rangle$ are SDs sampled by the QMC, and their probability distribution will give $w(\phi)(>0)$. Because the Schrödinger equation is linear, $\left|\Psi_{0}\right\rangle$ is degenerate with $-\left|\Psi_{0}\right\rangle$. A trivial effect in a deterministic representation, this can cause the determinants $|\phi\rangle$, in a random walk, to move back and forth between the two sets of solutions. In a simulation, precisely when a $|\phi\rangle$ turns from one to the other can not be detected, because the continuous stochastic evolution of the orbitals can lead to an exchange without any two orbitals ever overlapping. This is the sign problem. We use a trial wave function $\left|\Psi_{T}\right\rangle$ to make the detection, by requiring $\left\langle\Psi_{T} \mid \phi\right\rangle>0$. Because each $|\phi\rangle$ is a full many-electron wave function, the sign of its overlap with a $\left|\Psi_{T}\right\rangle$ is expected to be quite insensitive to the details of $\left|\Psi_{T}\right\rangle$.

In extensive benchmarks in Hubbard models [20, 21, 26] as well as in atoms, molecules, and solids [27], this general framework has demonstrated accuracy equaling or surpassing the most accurate (non-exponential scaling) many-body computational methods available. In the Hubbard model, the energy is typically within $<0.5 \%$ of the exact diagonalization results for $U=4 t$ [20]. At half-filling where the approximation is the most severe (with free-electron $\left|\Psi_{T}\right\rangle$ ), the method gives an energy per site of $-0.8559(4)$ for the infinite lattice, compared to the estimated exact result of $-0.8618(2)$ $[15,28]$. (The method can be made exact at half-filling by removing the constraint [21].)

In order to probe correlations at long range, we study rectangular supercells of $4 \times L_{y}, 8 \times L_{y}$ and $16 \times L_{y}$. The largest system size simulated in this work is $N \sim 1024$, using $\mathscr{O}(1000)$ processors on the Cray XT4 supercomputer. The first indication of a long wavelength collective mode is seen in the ground-state energy. Figure 1 shows how the energy per site, $\varepsilon$, varies as $L_{y}$ is increased. Each energy has been averaged over 20-1000 $\Theta$-values, and all controllable QMC biases (e.g., Trotter and population size [21]) have been removed. The error bars are estimated by combining statistical error and $\Theta$-point fluctuation. Twist-averaging eliminates kinetic energy finite-size effects (shell and lattice size) [20]. At $h=1 / 4$ for example, we have reconfirmed that the energy remained essentially constant as $L_{y}$ was varied from 8 to 64 .

At $h=1 / 16$, the energies remain above the line from Maxwell construction up to $L_{y} \sim 16$, where it shows a significant drop and falls below the line. In the inset, the equation of state (EOS), $\varepsilon(n)$ vs. $n$, is shown for $8 \times 8$. The EOS is concave for $n \in\left(n_{c}, 1\right)$ [20]. The critical density $n_{c}$ is determined by the Maxwell construction, which gives a phase separation line tangent to the EOS: $\varepsilon_{M}(n)=h / h_{c} \varepsilon\left(n_{c}\right)+\left(1-h / h_{c}\right) \varepsilon(n=$

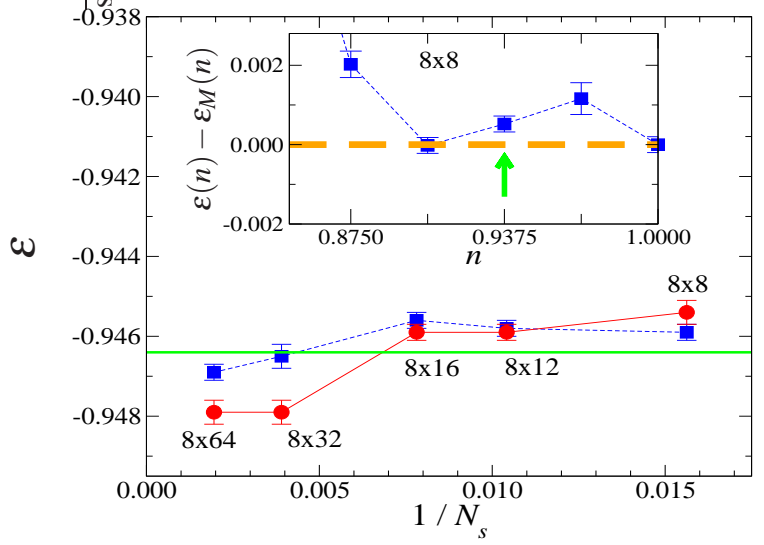

FIG. 1: Energy variations with linear dimension. The QMC ground state energy per site, $\varepsilon$ (in units of $t$ ), is shown for $U=4 t$ for a sequence of supercell sizes $8 \times L_{y}$. Blue squares are with an $\mathrm{FE}\left|\Psi_{T}\right\rangle$, while red circles are with a UHF $\left|\Psi_{T}\right\rangle$. The main graph is for $h=$ $1 / 16$. The green line is determined by Maxwell construction, which is illustrated in the inset. To magnify the vertical scale in the inset, a linear shift of $\varepsilon_{M}(n)$ has been applied, so the tangent line is horizontal (dashed orange), and the EOS is plotted as $\varepsilon(n)-\varepsilon_{M}(n)$.

1). The drop for $L_{y}>16$ indicates that the instability occurs only in smaller supercells, in which a state with long-range correlation is frustrated.

We use two different types of $\left|\Psi_{T}\right\rangle$, to help gauge the effect of the constraint. The first is the free-electron (FE) wave function, which is of course homogeneous with no long-range correlation. The second is the unrestricted Hartree-Fock (UHF) solution, which has broken spatial symmetry and static longrange spin and charge order. We have verified that, in the paramagnetic phase below $n_{c}$, the two types lead to statistically indistinguishable QMC results. As seen in Fig. 1, the same is true at $h=1 / 16$, except for large systems $\left(L_{y}>16\right)$, where the UHF $\left|\Psi_{T}\right\rangle$ gives lower energy. This is consistent with UHF being a better wave function in a system where an SDW can develop, as further discussed below.

We calculate the spin-spin correlation function:

$$
C_{S}(\mathbf{r})=\frac{1}{N} \sum_{\mathbf{r}^{\prime}}\left\langle\left(n_{\mathbf{r}+\mathbf{r}^{\prime}, \uparrow}-n_{\mathbf{r}+\mathbf{r}^{\prime}, \downarrow}\right)\left(n_{\mathbf{r}^{\prime}, \uparrow}-n_{\mathbf{r}^{\prime}, \downarrow}\right)\right\rangle,
$$

which measures the correlation between two spins separated by a lattice vector $\mathbf{r} \equiv\left(l_{x}, l_{y}\right)$. The corresponding structure factor is $S_{S}(\mathbf{k}) \equiv \sum_{\mathbf{r}} e^{i \mathbf{k} \cdot \mathbf{r}} C_{S}(\mathbf{r})$. Similarly, we calculate the charge-charge correlation $C_{c}(\mathbf{r})$, defined by replacing "-_" in Eq. (2) with “+”, and its structure factor $S_{c}(\mathbf{k})$.

The QMC results for $C_{S}(\mathbf{r})$ are shown in Fig. 2] An AF correlation is seen clearly in the density plots, in which the signs alternate for near neighbors. The staggered correlation function: $C_{s}^{\prime}\left(l_{x}, l_{y}\right) \equiv(-1)^{l_{y}} C_{s}\left(l_{x}, l_{y}\right)$, is also plotted with statistical error bars. The curves fall into two groups, for even and odd $l_{x}$, respectively. With perfect AF correlation, each group would be a constant function of $l_{y}$. In these systems the $\mathrm{AF}$ correlation patterns are modulated by a wave along $l_{y}$. A $\pi$ phase-shift occurs at the nodes where $C_{s}^{\prime}\left(l_{x}, l_{y}\right)$ crosses zero. 



FIG. 2: Spin-spin correlation function in $8 \times 32$ (top panel) and $8 \times 64$ (bottom panel) lattices at $h=1 / 16$ and $U=4 t$. The results are averaged over $20 \Theta$-points. UHF $\left|\Psi_{T}\right\rangle$ 's are used. The upper part in each panel is a 3D density plot (color theme in the upper right corner of the graph). The lower part of each panel shows the staggered correlation, with curves of different colors representing different $l_{x}$ 's. Due to symmetry, only $l_{y} \in\left[0, L_{y} / 2\right]$ is shown. The range of $C_{s}(\mathbf{r})$ is restricted to $[-0.1,0.1]$, so the "self-peak" near the origin is cut off.

The wave essentially doubles as $L_{y}$ is doubled, with comparable SDW amplitude. We see that the wavelength of the spin modulation is $\lambda \sim 32$ at this density, consistent with Fig. 1, where the energy lowering only occurs at $L_{y} \gg 16$.

We next study the spin-spin correlation as a function of doping. Calculations are done at three densities, $h=3 / 32,1 / 16$, and $1 / 32$, respectively, for a $4 \times 64$ lattice, at $U=4 t$. The staggered spin-spin correlation function $C_{s}^{\prime}(\mathbf{r})$ are shown in Fig. 3, where the modulation and $\pi$ phase shifts are clearly seen. The wavelength of the modulation decreases with doping, beginning at half-filling $(h=0)$ where the wavelength is $\lambda=\infty$. (Quantitatively, our data is consistent with the wavelength being inversely proportional to $h$, although statistical error bars on $\lambda$ are large.) The strength of $C_{s}^{\prime}(\mathbf{r})$ in the incommensurate SDW state also decreases with doping, and appears to vanish at a critical value of $h_{c} \sim 0.15 \pm 0.05$, where the system turns into a paramagnetic liquid.

The results in Fig. 2 2 were obtained using UHF $\left|\Psi_{T}\right\rangle$, while those in Fig. 3 were from $\mathrm{FE}\left|\Psi_{T}\right\rangle$. The consistency between them is reassuring. To generate the UHF $\left|\Psi_{T}\right\rangle$, we used the minimum $U$ for which a UHF solution exists $(U \sim 1.3-1.5 t$ for $h=1 / 16$ and $\sim 3.5 t$ for $h=1 / 4$ ), in order to minimize the effect of broken translational invariance. A weak static long-range order is present in the UHF solution. For example, in the $8 \times 64$ system, $C_{S}$ calculated from the UHF $\left|\Psi_{T}\right\rangle$ itself was $\mathscr{O}\left(5 \times 10^{-4}\right)$. We see that this was enhanced by a factor of 200 in the QMC, to $\mathscr{O}(0.1)$. On the other hand, at large distance the variation in the UHF charge-charge correlation $C_{c}$ was $\mathscr{O}\left(1.5 \times 10^{-4}\right)$, which remained $\mathscr{O}\left(10^{-4}\right)$ in the $\mathrm{QMC}$

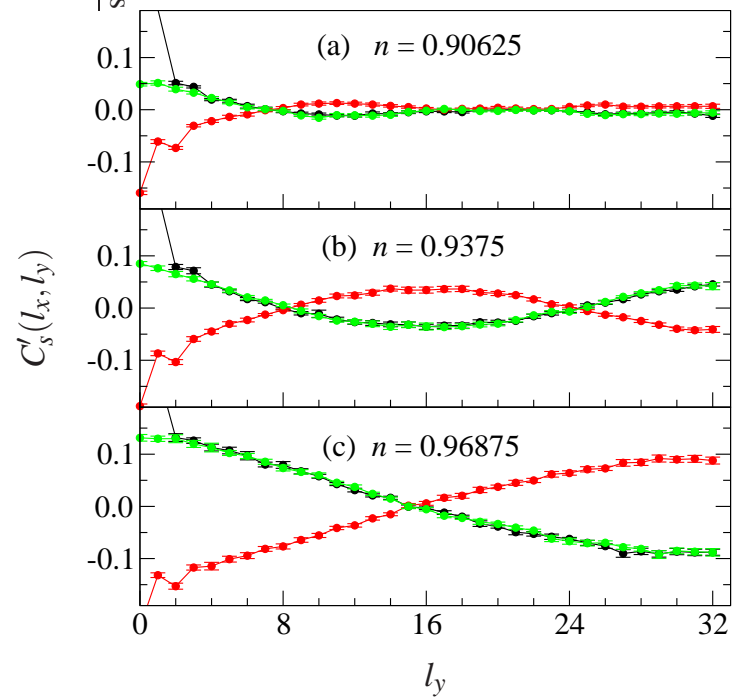

FIG. 3: Doping dependence of the long wavelength incommensurate SDW state. The staggered spin-spin correlation function $C_{s}^{\prime}\left(l_{x}, l_{y}\right)$ is plotted vs. $l_{y}$, at three different densities. Calculations are done using free-electron (FE) $\left|\Psi_{T}\right\rangle$. The system is a $4 \times 64$ supercell, with $U=4 t$ and $\Theta / \pi=(-0.8410,-0.9198)$. Colors label different value of $l_{x}$ 's as in Fig. 2 As doping is increased, the wavelength of the modulating wave decreases, as does the amplitude of the SDW.

(zero within error bars). With the FE $\left|\Psi_{T}\right\rangle$, the SDW structure in Fig. 3 emerged spontaneously. QMC results with UHF $\left|\Psi_{T}\right\rangle$ always showed long-range order, while we do find variations when the $\mathrm{FE}\left|\Psi_{T}\right\rangle$ is used: for some $\Theta$ 's no long-range SDW order is seen, only "short"-range incommensurate AF correlations. In such cases, the calculated QMC energy tends to be higher than that using UHF $\left|\Psi_{T}\right\rangle$. In contrast, when a long-range SDW is also seen with the $\mathrm{FE}\left|\Psi_{T}\right\rangle$, the QMC energies are more consistent between the two types of $\left|\Psi_{T}\right\rangle$ 's. We interpret this as the system favoring long-range order. The rectangular supercells break the symmetry between the $x$ - and $y$ - directions. In the thermodynamic limit a combination of the linear SDWs may be present.

The spin correlation discussed above $(U=4 t)$ is not accompanied by charge inhomogeneities. The effect of stronger interactions is examined in Fig. 4, which displays results for $U / t=4,8$ and 12 , with doping of $h=1 / 16$ in a $4 \times 32$ lattice. The spin structure factor $S_{s}(\mathbf{k})$ is plotted along the line cut $\mathbf{k}=\left(\pi, k_{y}\right)$. At $U=4 t$ a pronounced peak can already be seen at $(\pi, 15 \pi / 16)$, consistent with the spin-spin correlation in Fig. 2. (The split of the $S_{s}(\mathbf{k})$ peak with doping had also been observed in earlier simulations [3, 6, 10, 11].) As $U$ is increased, the peak value increases rapidly. The charge structure factor is plotted along $\left(0, k_{y}\right)$. Except for the trivial peak at the origin, $S_{c}(\mathbf{k})$ is broad with little features at $U=4 t$. Above $U=8 t$, a peak appears at $(0, \pi / 8)$, indicating the development of a charge-charge correlation.

In the inset of Fig. 4 the real-space density profile is plotted along $\left(0, l_{y}\right)$. At $U=4 t$, results using FE and UHF (generated with $U=1.4 t$ ) trial wave functions both give a constant density. At larger $U$, the same $\mathrm{UHF}\left|\Psi_{T}\right\rangle$ (from $U=1.4 t$ ) turned 


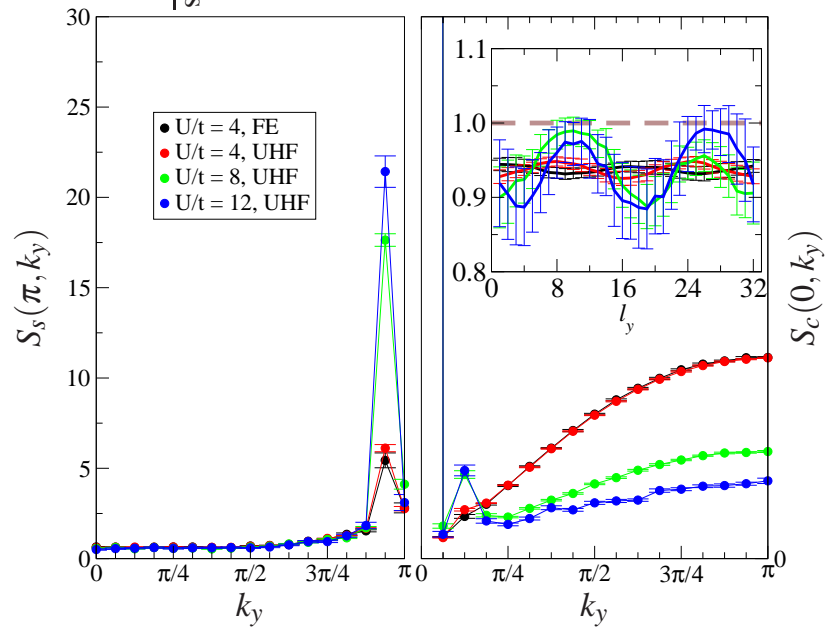

FIG. 4: Spin-spin and charge-charge structure factors as a function of interaction strength $U$. Results are shown for a $4 \times 32$ supercell, with $\Theta / \pi=(-0.8410,-0.9198)$ and at doping $h=1 / 16$. FE and UHF indicate the type of $\left|\Psi_{T}\right\rangle$ used in the QMC calculation. The inset in the right panel shows real space density profile with the same color coding as in the main figures. $S_{S}(\mathbf{k})$ has a peak at $\mathbf{k}=(\pi, 15 \pi / 16)$, with the peak value growing rapidly with increasing $U . S_{c}(\mathbf{k})$, which has no feature at $U=4 t$, shows a corresponding peak at $\mathbf{k}=(0, \pi / 8)$ for large $U$. The real-space density is a constant at $U=4 t$, but develops periodic modulation at larger $U$.

out to be sufficient to "pin" the many-body solution into a broken translational symmetry charge density wave state. The density profile provides a way to visualize the nature of the state. At $U=12 t$, the region of maximum density tends to saturate at $\rho=1$, while "stripes" appear at the boundaries which separate AF spin domains with a $\pi$ phase shift. This is consistent with density matrix renormalization group results [16] of stripe states in Hubbard ladders for $U \gtrsim 8 t$. The real-space characteristic length of the charge correlation is $1 / 2$ that of the spin correlation, as Fig. 4 shows. These results suggest that, at intermediate $U$, holes are in a uniform "liquid" state with no long-range correlation, while at the large $U$ limit they enter a "Wigner-crystal" state forming stripes.

The difficulty in treating the Hubbard model arises from the multiple competing energy scales separated by tiny differences. The system can fall into one phase or the other due to a small bias in the calculation or in simulation boundary condition. The challenge for numerical calculations is to minimize the effect of such biases (intrinsic accuracy, system size, etc). Our calculations reach much larger systems than possible otherwise. In this work, we have carefully removed biases other than the effect of the constrained path approximation. To address the latter, we have used trial wave functions with opposite properties (uniform FE vs. broken-symmetry UHF) to examine the robustness and consistency of the results.

To conclude, we have presented numerical results from constrained path QMC to characterize the magnetic properties in doped 2D Hubbard model. At intermediate interaction strengths $U / t \sim 4$, the ground state has incommensurate antiferromagnetic SDW order with long wavelength modulation.
The wavelength of the SDW and the strength of the spin order both decrease with doping, and the state vanishes below a critical density, when the system enters a paramagnetic "liquid" phase. In the SDW state there is essentially no charge correlation, with the holes in a wave-like state. As $U$ increases, accompanying charge correlation develops, with the holes becoming localized at the nodal positions of the modulating wave. Thus in the strong interaction regime $(U>\sim 10 t)$ the system evolves into a stripe-like state. Many topics remain for future work, including quantitative aspects of these states and their implications on superconductivity.

Computations were carried out at ORNL (Jaguar XT4) and William \& Mary (CPD and SciClone clusters). We thank Jie $\mathrm{Xu}$ and Eric Walter for help, and D. Ceperley, R. M. Martin, S. Sorella, and S. R. White for useful comments. This work is support by ARO (56693-PH) and NSF (DMR-0535592).

[1] M. Gutzwiller, Phys. Rev. Lett. 10, 159 (1963); J. Hubbard, Proc. R. Soc. London, A 276, 283 (1963); J. Kanamori, Prog. Theor. Phys. 20, 275 (1963).

[2] P.W. Anderson, Science 235, 1196 (1987);

[3] E. Dagotto, Rev. Mod. Phys. 66, 763 (1994); D.J. Scalapino, J. Low Temp. Phys. 95, 169 (2004).

[4] J.E. Hirsch, Phys. Rev. B 31, 4403 (1985); J.E. Hirsch and S. Tang, Phys. Rev. Lett. 62, 591 (1989);

[5] S.R. White, et al., Phys. Rev. B 40, 506 (1989).

[6] N. Furukawa and M. Imada, J. Phys. Soc. Jpn. 61, 3331 (1992).

[7] C.N. Varney et al., Phys. Rev. B 80, 075116 (2009).

[8] R. Jördens, et al., Nature 455, 204 (2008); U. Schneider, et al., Science 322,1520 (2008).

[9] M. Lewenstein, et al., Adv. Phys. 56, 243-379 (2007); I. Bloch, J. Dalibard, and W. Zwerger, Rev. Mod. Phys. 80, 885 (2008).

[10] M. Imada and Y. Hatsugai, J. Phys. Soc. Jpn. 58, 3752 (1989).

[11] A. Moreo, et al., Phys. Rev. B 41, 2313 (1990).

[12] H. Q. Lin, Phys. Rev. B 44, 7151 (1991).

[13] G. Fano, F. Ortolani, and A. Parola, Phys. Rev. B 46, 1048 (1992).

[14] A.C. Cosentini, et al., Phys. Rev. B 58, R14685 (1998).

[15] F. Becca, A. Parola, and S. Sorella, Phys. Rev. B 61, R16287 (2000); F. Becca, M. Capone, and S. Sorella, Phys. Rev. B 62, 12700 (2000).

[16] S.R. White and D.J. Scalapino, Phys. Rev. Lett. 91, 136403 (2003); G. Hager, et al., Phys. Rev. B 71, 75108 (2005).

[17] T. A. Maier, et al., Phys. Rev. Lett. 95, 237001 (2005).

[18] M. Aichhorn, et al., Phys. Rev. B 76, 224509 (2007).

[19] T. Aimi and M. Imada, J. Phys. Soc. Jpn. 76, 113708 (2007).

[20] Chia-Chen Chang and Shiwei Zhang, Phys. Rev. B 78, 165101 (2008).

[21] S. Zhang, J. Carlson and J. E. Gubernatis, Phys. Rev. Lett. 74, 3652 (1995); S. Zhang, J. Carlson, and J. E. Gubernatis, Phys. Rev. B 55, 7464 (1997).

[22] S. Zhang and H. Krakauer, Phys. Rev. Lett. 90, 136401 (2003).

[23] D. Poilblanc, Phys. Rev. B 44, 9562 (1991); C. Gros, Phys. Rev B 53, 6865 (1996); C. Lin, F. H. Zong, and D. M. Ceperley, Phys. Rev. E 64, 016702 (2001).

[24] H. Kwee, Shiwei Zhang, and Henry Krakauer, Phys. Rev. Lett. 100, 126404 (2008).

[25] Chia-Chen Chang and Shiwei Zhang, unpublished (2009). 
[26] W. Purwanto and S. Zhang, Phys. Rev. E 70, 056702 (2004); Phys. Rev. A 72, 053610 (2005).

[27] W. A. Al-Saidi, Shiwei Zhang, and Henry Krakauer, J. Chem. Phys. 124, 224101 (2006); ibid. 127, 144101 (2007); M. Sue- wattana, et al., Phys. Rev. B 75, 245123 (2007); [28] S. Sorella (private communication). 